\title{
Pile-tunnel interaction: A conceptual analysis
}

\author{
T.G.S. Dias \\ Ghent University, Ghent, Belgium
}

\author{
A. Bezuijen \\ Ghent University, Ghent, Belgium \\ Deltares, Delft, The Netherlands
}

\begin{abstract}
The underground space of densely populated cities contains parts of buildings, utility installations, deep foundations and tunnels. It is possible that new tunnels will be built within close proximity of existing pile foundations. Therefore pile tunnel interaction (PTI) must be assessed so that it is possible to ensure safety for both the tunnel construction and the pile-supported structures. This paper presents a simple model to evaluate the pile settlements and increment of axial force due to tunnel excavation. The displacement field around the tunnel can be used to estimate the pile displacement and the differential displacements around the pile-soil interface. Based on an example plane strain calculation of an unlined tunnel, different possible pile locations were evaluated. The results were compared to six studies from the literature and considerable agreement was obtained for the trends of ratios of pile to surface settlement and increment of axial stress on the pile.
\end{abstract}

\section{INTRODUCTION}

The underground space of densely populated cities contains parts of buildings, utility installations, deep foundations and tunnels. It is possible that new tunnels will be built within close proximity of existing pile foundations. Both structures might be located in the same soil layer to use the strength of a stiffer layer at greater depth. The pile tunnel interaction (PTI) must be assessed so that it is possible to ensure safety for both the tunnel construction and the pile-supported structures (Dias \& Bezuijen 2014).

The understanding of how the pile load-transfer mechanism is altered by the tunneling induced stress redistribution is still not completely developed. A considerable number of studies have evaluated the effects of new piles on existing tunnels and vice versa. However, Dias \& Bezuijen (2014) pointed out that the results of different physical models, by the parameters and layout evaluated, are not strictly reproducible. There are indications that the pile response depends on how much the shaft and tip resistance are mobilized and consequently if they can cope with the tunneling induced stress redistribution.

These studies normally present a relation between the soil displacements and the pile settlements. Kaalberg et al. (2005) and Selemetas et al. (2005) evaluated this relation by defining three zones around the tunnel, where pile settlements were larger (A), equal (B) or smaller $(C)$ than the surface settlements. How these zones are defined differed between the two studies as can be seen in Figure 1. On the other hand it can be argued that the pile settlements are better related to

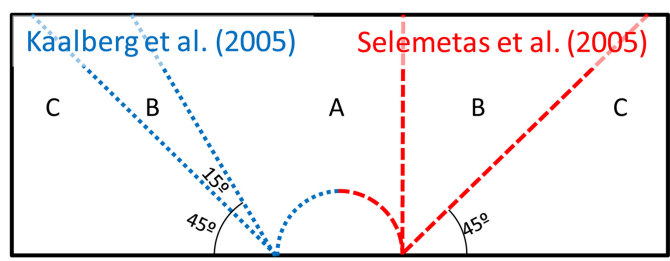

Figure 1. Zones of relative pile/soil settlement (modified from Kaalberg et al. (2005) and Selemetas et al. (2005)).

the soil settlements at some level along the pile depth. Mostly the tip level and 2/3 of the total depth are mentioned for this. (Devriendt \& Williamson 2011).

Based on these assumptions, this paper presents a very simple model to evaluate the pile settlements and increment of axial force due to tunnel excavation. The displacement field around the tunnel can be used both to estimate the pile settlement and calculate the differential displacements around the pile-soil interface. Assuming that no pile failure occurs, an elastic model relates these differential displacements to increments of axial force on the pile.

The parameters to define the pile-tunnel relative position are in Figure 2. Different interaction layouts will be mapped as in the methodology described by Dias \& Bezuijen (2014), which can be seen in Figure 3 . The depth of the tunnel and the pile are replaced by the difference between the tunnel springline depth $(\mathrm{Zt})$ and the pile length $(\mathrm{Zp})$. Both the vertical and the horizontal distances are normalized by the tunnel diameter (Dt). 


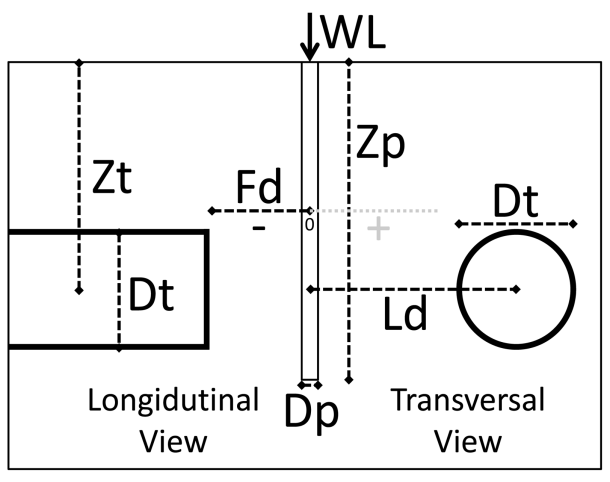

Figure 2. Parameters of the pile-tunnel relative positions.

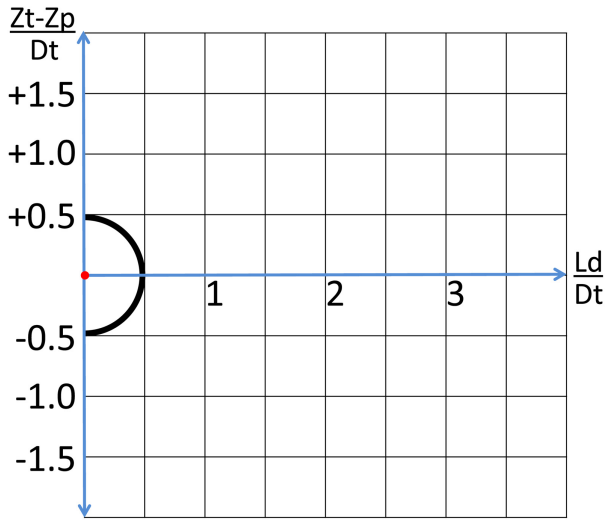

Figure 3. Pile-Tunnel interaction layout, lateral distance (Ld) and difference between the tunnel springline depth $(\mathrm{Zt})$ and the pile length $(\mathrm{Zp})$ normalized over the tunnel diameter (Dt).

\section{METHODOLOGY}

Assuming a certain displacement field around a tunnel, the settlements on a vertical section along the imaginary pile position, for example above the tunnel, can be determined ( $\delta_{s}$ on Figure 4). Following the argument that the pile settlements $\left(\delta_{p}\right)$ are equal to the soil settlements at some level along the pile depth, three possibilities are presented: at the surface, at $2 / 3$ of the pile depth and at the pile tip.

The pile is assumed to settle as a rigid body and does not follow the same settlement profile as the soil. At a certain depth, if the soil settlements are higher than the pile settlement, negative friction develops, increasing the axial force on the pile. If the soil settlements are smaller than the pile settlement, positive friction develops, reducing the axial force on the pile. Considering that in this example the pile settlement is derived from the soil settlements, at a certain depth the pile and the soil settlement will be equal. This position is normally referred as neutral plane and it is an imposition of this methodology.

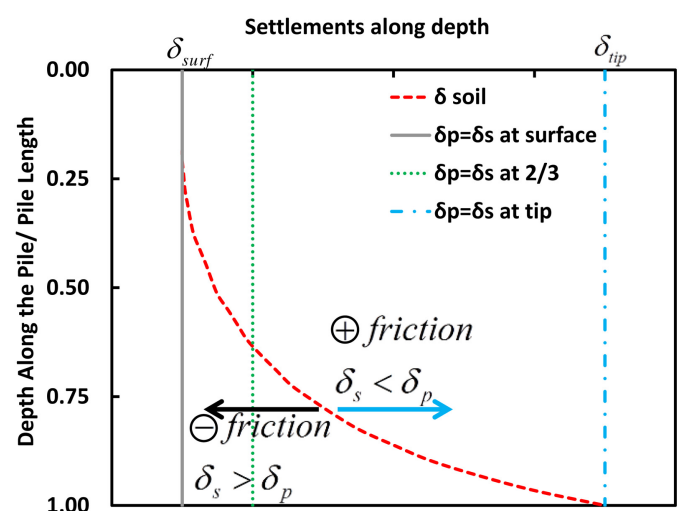

Figure 4. Example for pile-tunnel interaction analysis.

These incremental stresses can be added to the axial load distribution to verify that the ultimate pile capacity is not reached. This will, of course, depend on the maximum shear force that can develop on the pile-soil interface. This study assumes an elastic interface, but in reality once the shear resistance is achieved slippage will occur with no further increments.

Take for example the profile of Figure 4 and the assumption that the pile and soil settlements are equal at the level of the pile tip. In this case the soil settlements are smaller than the pile's along all its length and therefore only positive friction develops. On the other hand, in the case where the pile and soil settlements are equal at the surface, soil settlements are higher than the pile's along all its length and only negative friction develops. For the intermediate case, where the pile and soil settlements are equal at the level of $2 / 3$ the pile depth, both positive and negative friction develops. On the top $2 / 3$ of the pile the soil settles less than the pile inducing positive friction. On rest of the pile the soil settles more, inducing negative friction (Fig. 4).

Even from numerical methods the soil settlements are normally known in a discrete number of points along the section. The increment of axial stress $\left(\Delta \sigma_{\mathrm{AS}}\right)$ between two points $(n, n+1)$ can be determined by the average difference between the soil and the pile settlement $(\delta)$. This average is then divided by the pile length between the two points that will results in the average interface shear strain. Considering a certain shear modulus $(\mathrm{G})$ for the interface the shear stress can be directly determined. If that stress is integrated along the interface surface and divided by the pile area the increment of axial stress can be determined using Equation 1.

$\Delta \sigma_{A S}=\left[\left(\delta_{s}^{n}-\delta_{p}\right)+\left(\delta_{s}^{n+1}-\delta_{p}\right)\right] \frac{2 \cdot G}{D_{p}}$

This equation calculates the increment of axial force caused by the imposed boundary condition of pile settlement. The tunnel induced displacements were obtained from a drained plane-strain finite element 


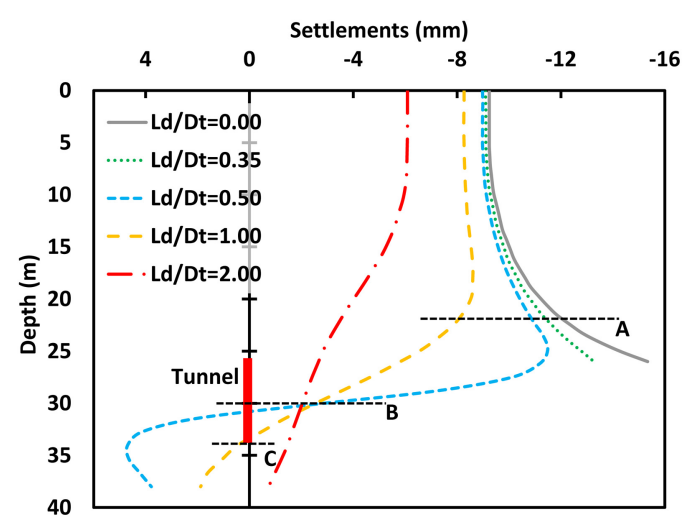

Figure 5. Settlement profile around the tunnel.

analysis of an unlined tunnel with a stress release factor $\lambda=0.50$, which corresponds for the soil conditions mentioned below to a volume loss of $0.42 \%$ on the tunnel level and $0.91 \%$ on the surface. The tunnel springline was at a depth of $30 \mathrm{~m}$ and the tunnel diameter was $8 \mathrm{~m}$. The soil was modelled by the Hardening Soil with small-strain stiffness model. The parameters were estimated from the empirical correlations of Brinkgreve et al. (2010) for sand at a relative density of 0.75 and are namely: $\phi=37^{\circ} ; \psi=7^{\circ}$; $v=0.2 ; \mathrm{m}=0.466 ; \mathrm{E}_{50}^{\text {ref }}=45 \mathrm{MPa} ; \mathrm{E}_{\text {oed }}^{\text {ref }}=45 \mathrm{MPa}$; $\mathrm{E}_{\text {ur }}^{\text {ref }}=135 \mathrm{MPa} ; \mathrm{G}_{0}^{\text {ref }}=111 \mathrm{MPa} ; \gamma_{0.7}=1.25 \cdot 10^{-4}$. The parameters $\mathrm{k}_{0}=0.50$ and $\gamma=20 \mathrm{kN} / \mathrm{m}^{3}$ were adopted. For the complete formulation of the model the reader is referred to Brinkgreve et al. (2013).

The vertical displacements were assessed on sections positioned at a lateral distance $(\mathrm{Ld} / \mathrm{Dt})$ of $0,0.35$, $0.50,1.00$ and 2.00 tunnel diameters from the tunnel centerline and shown in Figure 5. Three pile lengths were analyzed: (A) 22; (B) 30 and (C) $34 \mathrm{~m}$. Pile A was analyzed on $\mathrm{Ld} / \mathrm{Dt}$ values of $0,0.35,0.50$ and 1.00 . Pile B on Ld/Dt $0.50,1.00$ and 2.00 and Pile $C$ on $\mathrm{Ld} / \mathrm{Dt} 1.00$.

In all analyzes the piles were assumed to settle the same as the soil of their tip level, as is marked on Figure 5. The interface shear modulus was assumed $\mathrm{G}=20 \mathrm{MPa}$ and the piles were all $1 \mathrm{~m}$ in diameter. Following the layout of Figure 3 the piles can be positioned according to Figure 6. Point A0.35, for example, refers to a $22 \mathrm{~m}$ long pile, whose tip is one tunnel diameter above the tunnel springline and 0.35 tunnel diameters to the side of the tunnel center.

The results of this conceptual model will be compared to six other studies that were reported by Dias \& Bezuijen (2014) and will be briefly described hereafter.

Kaalberg et al. (2005) reported the results of a site test that was prepared along the construction of the 2nd Heinenoord tunnel in The Netherlands to investigate pile tunnel interaction for future tunneling construction in Amsterdam. Above the twin TBM tunnels, clay columns were created in the sand to reproduce the typical soil profile and the wooden end bearing piles

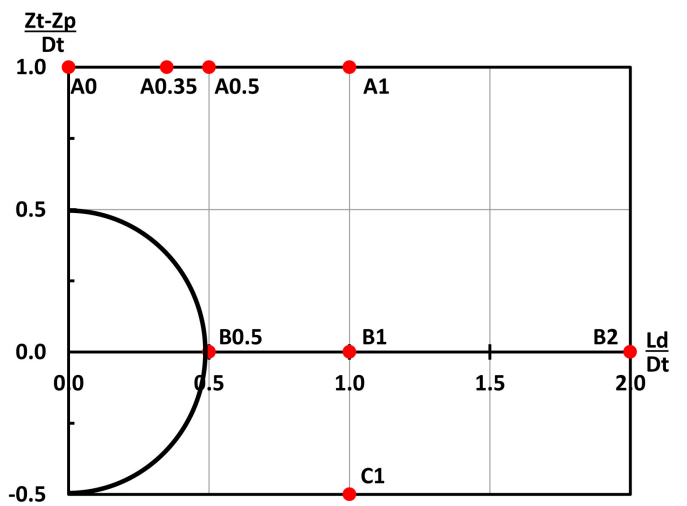

Figure 6. Pile's tip positions around the tunnel.

of the city. Another test site was prepared in the UK along the construction of the new Channel Tunnel Rail Link (Selemetas et al. 2005). Friction and end bearing driven piles, loaded to $50 \%$ their ultimate bearing capacity, were monitored during the construction of twin EPB tunnels.

It is not always feasible to perform full scale field tests, therefore reduced scale physical models are also an important source of data on pile tunnel interaction, especially centrifuge tests. Bezuijen \& Van der Schrier (1994) performed centrifuge tests with a model tunnel whose diameter could be adjusted by a wedge between the internal core and the external lining. The typical Dutch profile of soft clay over sand and driven piles was analyzed. The pile tip and the tunnel were based on the sand layer. Jacobsz et al. (2004) employed a model tunnel of a rigid cylinder enveloped by a rubber membrane. The volume of the water that filled the gap between the inner core and the membrane was reduced to simulate the tunnel volume loss. The model piles were jacked $2 \mathrm{~m}$ in flight on a sand profile. Feng et al. (2002) used a model tunnel composed of high density polystyrene foam that was dissolved in flight inside a brass foil and model piles already in place for the sand pouring. Lee and Chiang (2007) modeled the tunnel controlling the air pressure inside a thick cylindrical rubber bag on which a sheet of filament tape was pasted. The piles were already in place during sand pouring.

\section{RESULTS AND DISCUSSION}

According to the methodology exposed the pile settlements (equal the soil settlement at the pile tip level) were calculated and compared to the soil surface settlements for the different lengths and positions. Figure 7 presents the results of pile and surface settlements and their ratio.

All the A piles settle more than the surface, with the exception of Pile A1, where the pile/surface settlement ratio is slightly smaller than 1 . Piles $B$ and $C$ settles less than the soil at surface. As the lateral distance increases for Piles B, the magnitude of settlements 


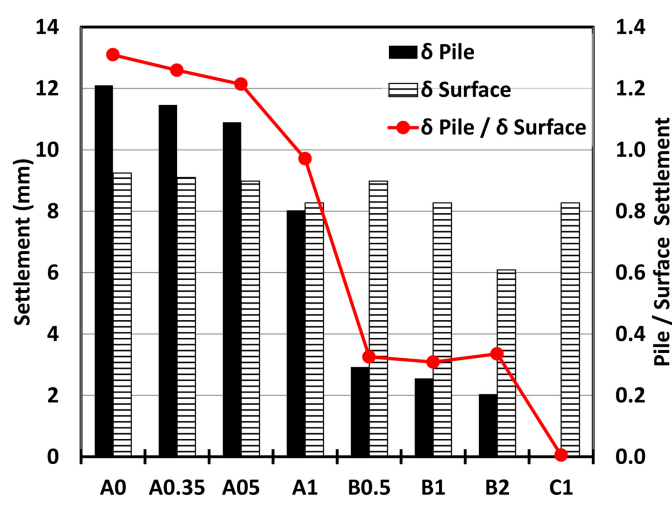

Figure 7. Pile/Surface settlement ratio for the different piles of the analysis.

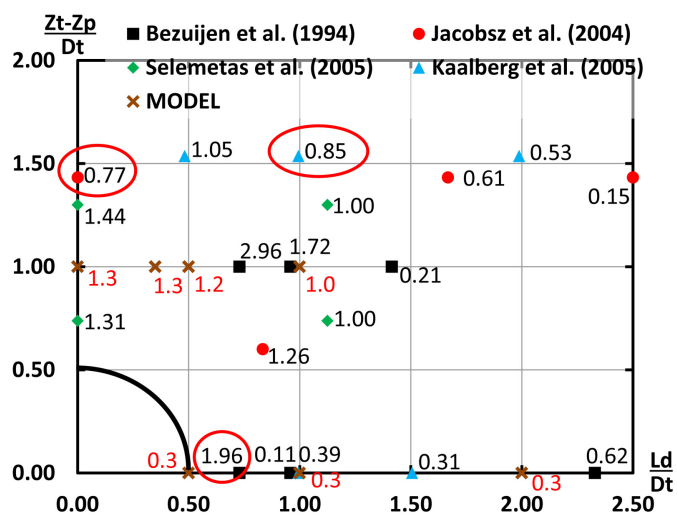

Figure 8. Pile/Surface settlement ratio calculated and reported on literature.

decrease but the settlement ratio remains roughly constant. Pile $\mathrm{C} 1$ presents no settlements, as its tip position matches the depth where the soil displacements at that lateral position changed from negative to positive.

Figure 8 presents the ratio of pile and surface settlement together with the results of four studies that reported the values of pile and surface settlements. The points are marked on the layout of Figure 3 together with the ratio value. The calculated values are in red to be distinguished from the measured values.

Where $\mathrm{Ld} / \mathrm{Dt}$ is higher than 1 , all the literature data agrees with the model, as the settlement ratios are smaller or equal to 1 . For $\mathrm{Ld} / \mathrm{Dt}$ between 0.5 and 1.0 the model implied that the settlement ratios would be higher than unity for piles tips above the tunnel and smaller than unity close to the tunnel springline. Most results agree with that, but there are two points in disagreement. The point with a ratio of 0.85 probably was under a different profile of soil displacement due to different soil conditions. The other point is likely to have failed as it was very close to the tunnel and under a load equivalent to $70 \%$ its capacity. Directly above the tunnel, the data from Selemetas et al. (2005) agrees with the model prediction of higher pile settlements,

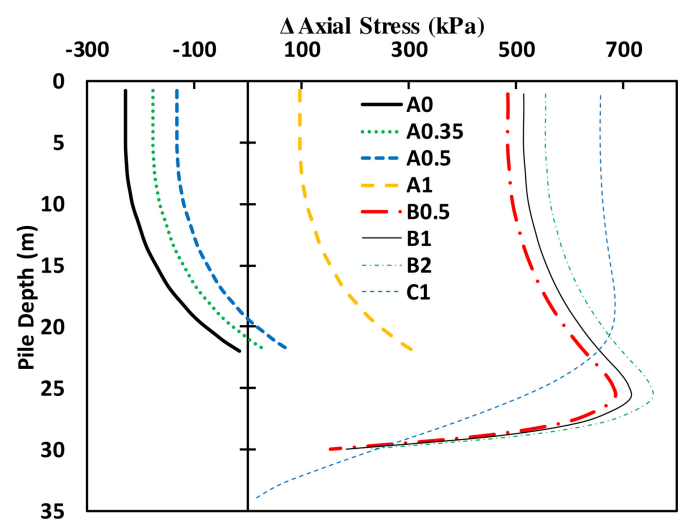

Figure 9. Increment of axial stress on the different piles of the analysis.

but the results from Jacobsz et al. (2004) present a settlement ratio below 1 . All the points in disagreement with the predictions are circled in Figure 8.

Based on the settlement profiles (Fig. 5) the increment of axial stress on the piles (Fig. 6) was calculated according to Equation 1, the results are shown in Figure 9. As it was, the increment of axial stress is a direct consequence of interface shear modulus and the relation between the soil settlement profile and the determined pile settlement. The piles that settled more than the soil settlements at surface (A0, A0.35 and A0.5) have developed positive friction along most of their sections that resulted in a decrease in their axial force. The stress increments decrease with depth as the soil settlements approach the value of the pile settlement. The gradient of axial stress is directly connected to the gradient of the soil settlements along the pile depth.

Based on the settlement profiles (Fig. 5) the increment of axial stress on the piles (Fig. 6) was calculated according to Equation 1, the results are shown in Figure 9. As it was, the increment of axial stress is a direct consequence of interface shear modulus and the relation between the soil settlement profile and the determined pile settlement. The piles that settled more than the soil settlements at surface (A0, A0.35 and A0.5) have developed positive friction along most of their sections that resulted in a decrease in their axial force. The stress increments decrease with depth as the soil settlements approach the value of the pile settlement. The gradient of axial stress is directly connected to the gradient of the soil settlements along the pile depth.

On the other hand, piles that presented settlement ratios below unity developed negative increments of axial stress over most of its depth. All piles that were $30 \mathrm{~m}$ deep (B) presented similar stress profiles, with an inflexion at the depth when the difference between the pile and the soil settlements start to decrease.

The results of three studies that reported increments of axial force due to tunnel construction are presented in Figure 10. Those values are not to be 


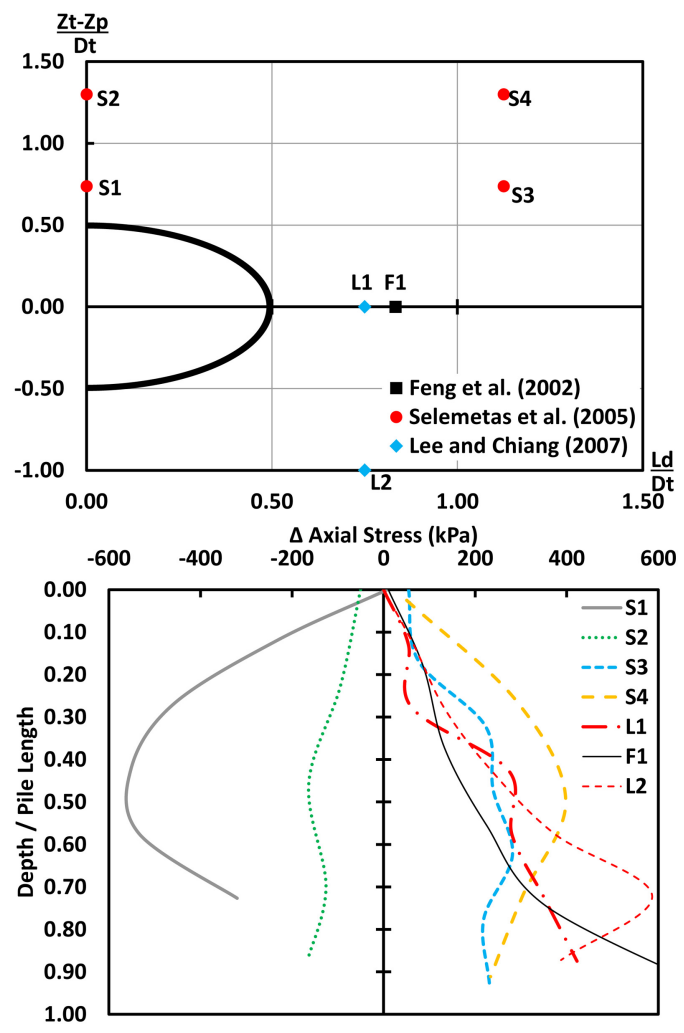

Figure 10. Increment of axial stress from literature.

compared quantitatively with the results of our calculations, which assumed an arbitrary interface shear modulus and are not bounded by load boundary conditions. However, the general response of increase of decrease in axial stress can be compared.

On the top of the tunnel, there was also a decrease in the axial stress on the pile. The gradient however, was quite different. The results of the calculation model indicate a steady decrease in the axial force increment, which was not at all present for Pile S2 and just evident on the last $1 / 3$ of Pile S1. Pile S3 agreed roughly with Pile A 1 from the model. Pile S4 presented a decrease in the axial stress that was not evident in the model. Piles L1 and F1 had positive axial stress increments along all its depth. Pile L2 presented the expected inflexion, but on a deeper level than pile $\mathrm{C} 1$ from the model.

\section{CONCLUSION}

A simple model for the analysis of tunneling induced pile settlements and the consequent increment of axial stress was presented. The model is a first step to understand how a pile can interact with the displacement field around a tunnel.

Based on an example plane strain calculation of an unlined tunnel, different possible pile locations were evaluated. The results were compared to six studies from the literature and considerable agreement was obtained for the trends of ratios of pile to surface settlement and increment of axial stress.

Some features of the pile response that were normally associated with complex changes on the pile tip and shaft load-transfer mechanism could be captured based on a simple relation between pile and soil settlements.

\section{ACKNOWLEDGEMENTS}

The first author would like to acknowledge the financial support of "Conselho Nacional de Desenvolvimento Científico e Tecnológico-CNPq, Brasil".

\section{REFERENCES}

Bezuijen, A. \& van der Schrier, J.S. 1994. The influence of a bored tunnel on pile foundations. In: C. F. Leung, F. H. Lee \& T. S.Tan (eds), International Conference Centrifuge 94, Singapore.

Brinkgreve, R.B.J., Engin, E. \& Engin, H.K. 2010. Validation of empirical formulas to derive model parameters for sands. In: T. Benz \& S. Nordal (eds), 7th European Conference on Numerical Methods in Geotechnical Engineering - NUMGE 2010, Trondheim.

Brinkgreve, R.B.J., Engin,E. \& Swolfs, W.M. 2013. Plaxis 2D 2012 Material Models Manual. Plaxis BV, Delft.

Burland, J.B. \& Wroth, C.P. 1974. Settlements of Buildings and Associated Damage. In, Conference on Settlement of Structures, Cambridge.

Devriendt M. \& Williamson M. 2011. Validation of methods for assessing tunnelling-induced settlements on piles. Ground Engineering Magazine, March, 25-30.

Dias, T.G.S. \& Bezuijen, A. 2014. Pile Tunnel Interaction: Literature Review and Data Analysis. In, World Tunnel Congress 2014 - Tunnels for a better Life, Foz do Iguaçu, Brazil.

Feng, S.H., Leung, C.F., Chow, Y.K. \& Dasari, G.R. 2002. Centrifuge modelling of pile responses due to tunnelling. In: The 15th KKCNN Symposium on Civil Engineering, Singapore.

Jacobsz, S.W., Standing, J.R., Mair, R.J., Soga, K., Hagiwara, T. \& Sugiyama, T. 2004. Centrifuge modelling of tunnelling near driven piles. Soils and Foundations 44(1), $51-58$.

Kaalberg, F.J., Teunissen, E.H., van Tol, A.F. \& Bosch, J.W. 2005. Dutch research on the impact of shield tunnelling on pile foundations, In: K.J. Bakker, A. Bezuijen, W. Broere \& E.A. Kwast (eds), International Symposium on Geotechnical Aspects of Underground Construction in Soft Ground, Amsterdam.

Lee, C.J. \& Chiang, K.H. 2007. Responses of single piles to tunnelling-induced soil movements in sandy ground. Canadian Geotechnical Journal 44 (10), 1224-1241.

Selemetas, D., Standing, J.R. \& Mair, R.J. 2005. The response of full-scale piles to tunnelling, In: K.J. Bakker, A. Bezuijen, W. Broere \& E.A. Kwast (eds), International Symposium on Geotechnical Aspects of Underground Construction in Soft Ground, Amsterdam. 